\title{
Storing and Harvesting Atoms/Molecules On-Chip: Challenges and Applications
}

N. Thammawongsa, S. Tunsiri, M.A. Jalil, J. Ali and P. P. Yupapin*

Department of Physics, King Mongkut's Institute of Technology, Thailand

Behaviors of light in nano/micro optical devices have shown the promising results of light which can be useful for many fundamental and applied researches, especially, in nano-electronics. Pornsuwancharoen and Yupapin [1] have proposed the use of a simple device called "microring resonator" to perform such behaviors. In this research work, the four different behaviors of light i.e. fast, slow, stopping and storing of light are investigated using a ring resonator. Nowadays, stopping or cooling light beam has become the promising technique for atom/molecule trapping investigations (using static or dynamic tweezers), especially, after the announcement of Nobel Prize 2012 in Physics on the whispering gallery modes [2,3]. There are two more kinds of devices that can be used to trap light beams, the use of microcavity arrays performed by Yanik and Fan [4], and nonlinear microring resonator by Yupapin and Pornsuwancharoen [5] for stopping light (laser beam). Ang and Ngo have also done experiment to slow the light in microresonators using a microring system recently [6]. This concept is a concrete backbone for many applications.

In this letter, a new design of microring resonator device is proposed which can be used to generate four forms of light simultaneously on a chip, while the storing and harvesting of trapped atoms/molecules can also be available. The proposed device is made up of silica and InGaAsP/ InP with linear optical add-drop filter incorporating two nonlinear micro/nano rings on both sides of the center ring (add-drop filter). This particular configuration is known as a "PANDA" ring resonator [7] as shown in figure1. Light pulse, for instance, Gaussian, bright and dark solutions are fed into the system through different ports such as add port and through port. By using the practical device parameters, the simulation results are obtained using the Opt wave and MATLAB programs. Results obtained by both analytical and numerical methods show that many applications can be exploited. In application, when the practical device parameters are used, then such device can be fabricated and implemented in the near future.

The proposed system can be used for many applications, especially, when the device is coated by a metallic material [8]. The applications such as atom/molecule transportation, molecule/atom antenna, atomic/ molecular automata, atom/molecule electronic devices, cells/atom distributed sensors, cells/atom radio, cells communications, micro Faraday cage, molecular/atom storage and logistics, atom/molecule trapping (storage) or cooling, everlasting atom/molecule investigation atomic/molecular storage for quantum gate or computer application,

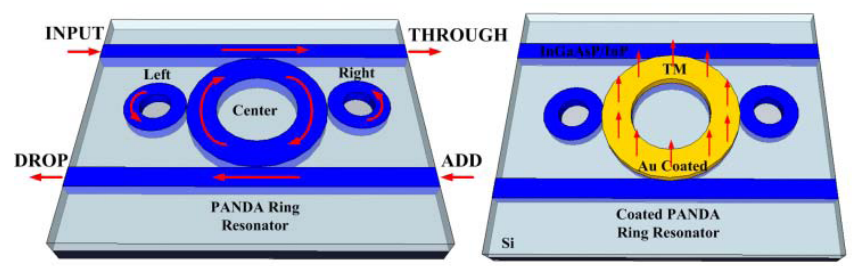

Figure 1: A conventional PANDA ring planar wave guide was named and designed by Uomwech et al [7] and Gold coated PANDA ring resonator for TM polarized coupling device. storm search and navigator sensors, magnetic/spin networks, magnetic net, micro plasma source, 3-D flat panel device and large cooling area (volume) can be constructed in the same way as a single device. In this work one of them is demonstrate. Under the stopping and storing condition, the system is considered as the storage unit, where atom or molecule can be trapped by the whispering gallery light beam at the center.

The whispering gallery mode result is obtained by using the Optiwave program as shown in figure 2. The ring material is InGaAsP/ InP, where the device parameters are given in figure caption. By using the MATLAB program, the whispering gallery modes of four state of light i.e. fast, slow, stopping and storing can be generated and controlled simultaneously on-chip as shown in figure 3 . The storing stage can be seen easily, while the stopping condition can be observed and the following conditions satisfied: (i) the center signal is lost in time between fast and slow signals or (ii) there is no movement among trapped particles or molecules i.e. the exchange of angular momentum introduces the conservation of angular momentum, where the combination of scattering and gradient forces is balanced under the adiabatic process.

The stopping light in term of signal condition can be easily performed using the whispering gallery mode concept, where the fast and slow light can be used as the upper and lower time frames or upper

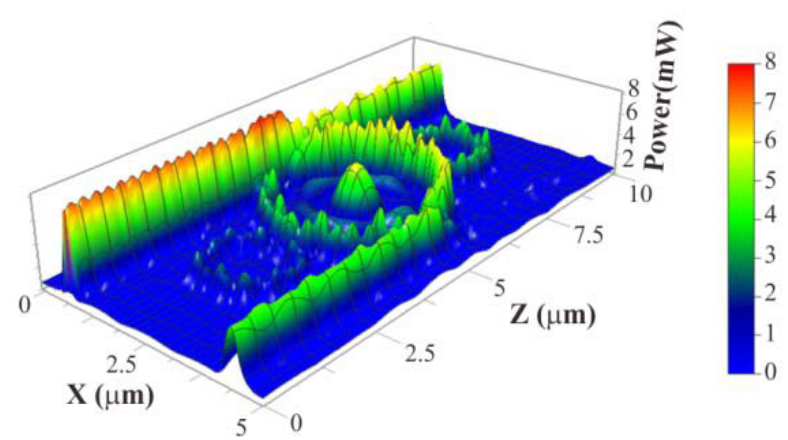

Figure 2: Result of whispering gallery mode of light within a PANDA, waveguide InGaAsP/InP, $\mathrm{R}_{1}=\mathrm{R}_{2}=0.775 \mu \mathrm{m}, \mathrm{R}_{\mathrm{ad}}=1.565, \mathrm{~A}_{\text {eff }}=0.3 \mu \mathrm{m}^{2}, \mathrm{n}_{\text {eff }}=3.14, \mathrm{n}_{2}=1.3$ $\times 10^{-13} \mathrm{~cm}^{2} / \mathrm{W}, \kappa_{1}=\kappa_{2}=\kappa_{3}=\kappa_{4}=0.5, \gamma=0.01, \lambda_{0}=1,550 \mathrm{~nm}$.

${ }^{*}$ Corresponding author: Preecha P Yupapin, Professor, Department of Physics, King Mongkut's Institute of Technology, Thailand; E-mail: kypreech@kmitl.ac.th

Received November 05, 2012; Accepted November 06, 2012; Published November 08, 2012

Citation: Thammawongsa N, Tunsiri S, Jalil MA, Ali J, Yupapin PP (2012) Storing and Harvesting Atoms/Molecules On-Chip: Challenges and Applications. J Biosens Bioelectron 3:e114. doi:10.4172/2155-6210.1000e114

Copyright: ( 2012 Thammawongsa N, et al. This is an open-access article distributed under the terms of the Creative Commons Attribution License, which permits unrestricted use, distribution, and reproduction in any medium, provided the original author and source are credited. 
Citation: Thammawongsa N, Tunsiri S, Jalil MA, Ali J, Yupapin PP (2012) Storing and Harvesting Atoms/Molecules On-Chip: Challenges and Applications. J Biosens Bioelectron 3:e114. doi:10.4172/2155-6210.1000e114
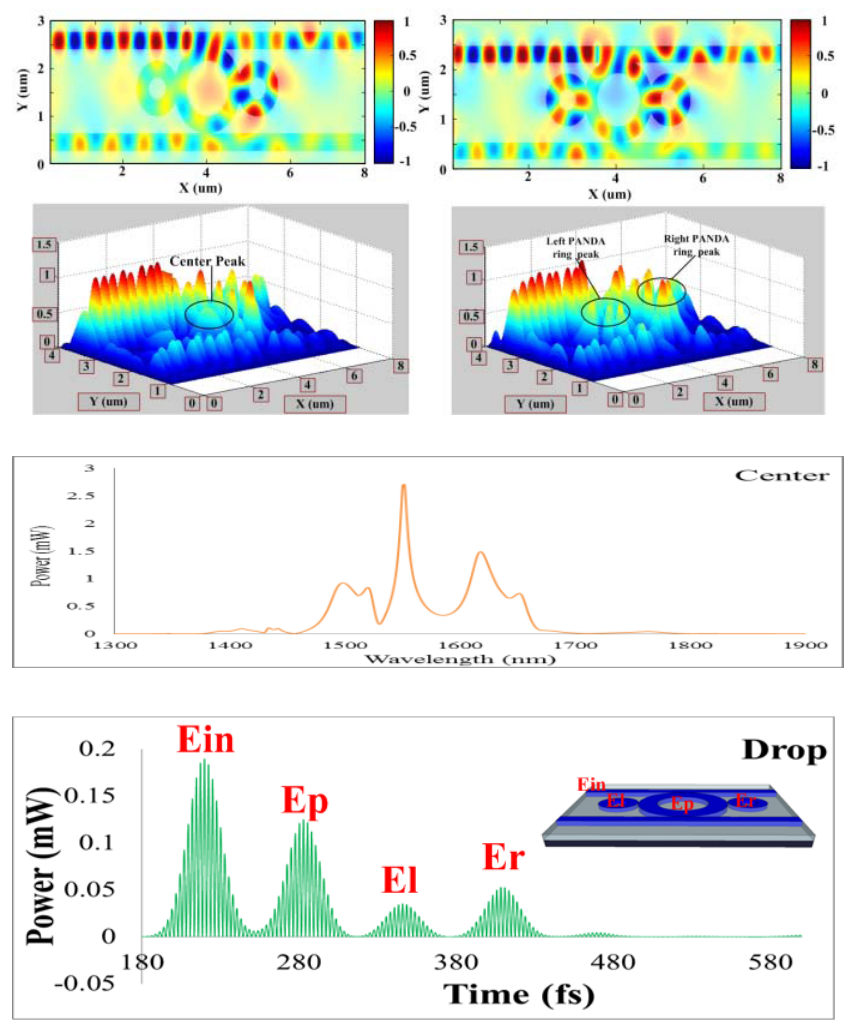

Figure 3: Stopping and storing light simultaneously detected using a PANDA ring, where (a) Center ring, (b) Side rings, (c) Center peak and side peaks, (d) Fast $\left(2^{\text {nd }}\right.$ peak) and slow ( $4^{\text {th }}$ peak) light detected at drop port with time interval of $150 \mathrm{fs}$.
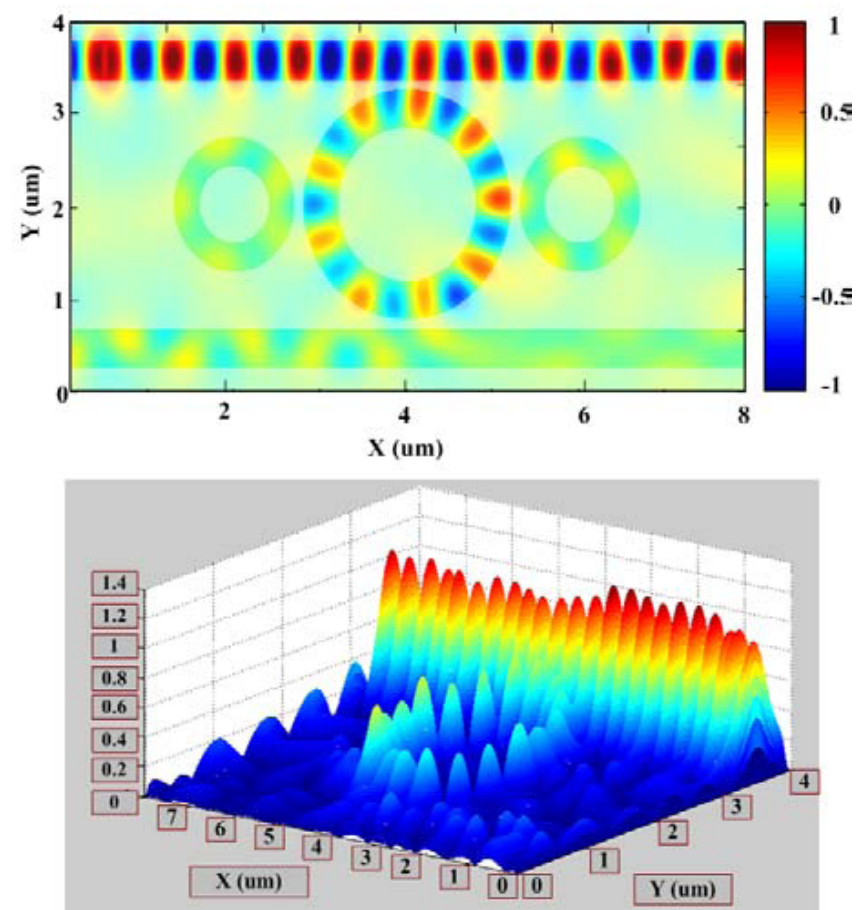

Figure 4: Dynamic tweezers are generated by a PANDA ring and transmitted via a Through port for atoms/molecules harvesting and transportation.

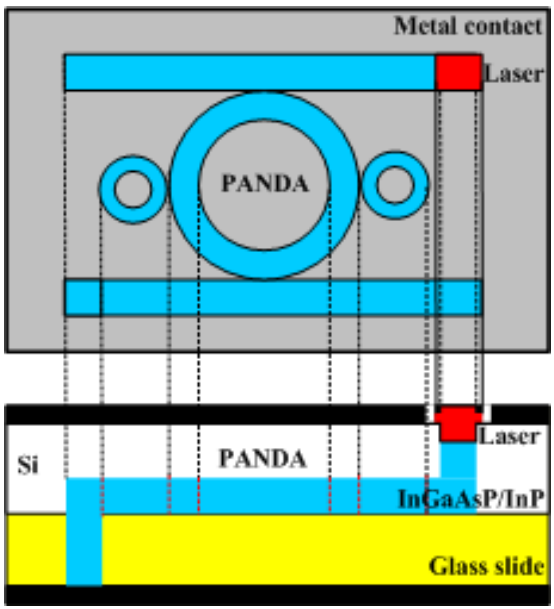

Figure 5: New type solar cells using a PANDA and embedded particle accelerator (Optical trapping and transportation)

side and lower side peak signals for the storing light at the center as shown in figure 3 , where in this case the movement (modulated signals) longer than 150 fs, i.e. ms, ns, ps is observed (stopped). The input pulse is a Gaussian pulse with pulse width of $100 \mathrm{fs}$, the fast and slow time interval is known, however, the whispering gallery modes can be seen only under the resonant condition.

The use of light trapping probe for atom/molecule trapping and transportation (dynamically trapping) can be formed with wide range of applications. In this case the modulated signal is required to switch off the whispering gallery mode power via the add port, where finally the trapped atoms/molecules at the device center can be trapped and transported along the wave guide by the surface plasmon tweezers as shown in figure 4 . The dynamic tweezers are generated by a PANDA ring, where in practice, particle angular momentum can be introduced by a metal coating material on the waveguide surface or combining the external modulation via the add port, which can be used to trap and transport atom/molecule to the required destination when the gradient force is greater than the scattering force along the waveguide. For example, the use of such concept for new type solar cells is as shown in figure 5, where free electrons from the depletion region can be trapped and transported(injected) to the metal contact faster than the conventional device, the solar cells efficiency can be increased by 5 times to the conventional one [9].

\section{References}

1. Pornsuwancharoen N, Yupapin PP (2009) Microw \& Opt. Techn. Lett 51: 899 902.

2. Wineland DJ, Bollinger JJ, Itano MW, Prestage JD (1985) JOSA B 2: 1721 1730 .

3. Knight JC, Dubreuil N, Sundoghdar V, Hare J, Lefevre-Seguin V, et al. (1996) Opt. Lett., 21: 698-670.

4. Yanik MF, Fan S (2004) Phys. Rev. Lett 92: 083901-083903.

5. Yupapin PP, Pornsuwancharoen N (2009) IEEE Photon. Techn. Lett 21: 404 406

6. Ang TYL, Ngo NQ (2012) Tunable flat-band slow light via contra-propagating cavity modes in twin coupled microresonators. JOSA B 29: 924-933.

7. Uomwech K, Sarapat K, Yupapin PP (2010) Microw. \& Opt. Techn Lett 52 1818-1821.

8. Thammawongsa N, Moonfangklang N, Mitatha S, Yupapin PP (2012) PIER L, 31: $75-87$.

9. Ueamanapong SS, Niemcharoen S, Yupapin PP (2012) Optics Express 20 12640-12648. 